\begin{tabular}{|c|c|c|c|}
\hline Nr. & $\begin{array}{c}\tau_{0} \text { vor der } \\
\text { Bestrahlung } \\
{\left[\mathrm{p} / \mathrm{mm}^{2}\right]}\end{array}$ & $\begin{array}{l}\text { Abgleitung } \\
\text { vor der } \\
\text { Bestrahlung }\end{array}$ & $\begin{array}{c}\tau_{0} \text { nach Bestr. mit } \\
9,6 \cdot 10^{17} \mathrm{nvt} \\
{\left[\mathrm{kp} / \mathrm{mm}^{2}\right]}\end{array}$ \\
\hline 1 & 58 & 0,004 & 2,45 \\
\hline 2 & 67 & 0,006 & 2,59 \\
\hline 3 & 94 & 0,006 & $\pm 1,5 \%$ \\
\hline 4 & 192 & 0,006 & 2,46 \\
\hline 5 & 257 & 0,006 & 2,46 \\
\hline
\end{tabular}

Tab. 2. Kritische Schubspannung bei $294^{\circ} \mathrm{K}$.

Die unterschiedlichen $\tau_{0}$-Werte nach der Bestrahlung hängen also nicht mit denen vor der Bestrahlung zusammen. Sie sind u. E. durch den Flußgradienten im Reaktor bedingt; die Kristalle Nr. 1, 4 und 5 lagen in einer Aluminiumhülse, die Kristalle Nr. 2 und 3 in einer anderen. Beide wurden dann nebeneinanderstehend bestrahlt. Eine derartige Gruppenbeziehung zwischen KSS und Bestrahlungsort trat bis auf zwei Ausnahmen bei allen 48 Kristallen auf. In einem anderen Versuch wurden je drei Kristalle in zwei Aluminiumhülsen bestrahlt und zwischendurch so vertauscht, daß sich ein even- tueller Flußgradient herausmitteln sollte. Alle sechs Kristalle zeigten danach die gleiche $( \pm 1,5 \%)$ KSS. $\mathrm{Ob}$ allerdings Streuungen, wie sie in Abb. 10 bei $2 \cdot 10^{18}$ nvt zu erkennen sind, nur durch diesen Einfluß entstanden sind, erscheint uns fraglich und bedarf weiterer Untersuchungen.

Meinem verehrten Lehrer, Herrn Prof. W. Rollwagen, danke ich für die Möglichkeit zu dieser Arbeit und für seine ständige Bereitschaft, Hilfe zu leisten. - Mein besonderer Dank gilt Herrn Dr. Christoph Schwink für die Anregung zu dieser Arbeit, für seine rege Anteilnahme und viele fördernde Diskussionen. - Von der Reaktorstation in Garching gilt mein Dank besonders Herrn Dr. W. Marth und Herrn Dr. W. Köhler für die Ausführung der Bestrahlungen und wertvolle Hinweise. - Durch die freundliche Genehmigung von Herrn Prof. Dr. W. Meissner und der Bayer. Akademie der Wissenschaften konnte uns Herr Dr. W. W Iedemann mit flüssigem Wasserstoff versorgen. Ihnen danke ich für diese Hilfe. - Die Arbeit wurde dankenswerterweise vom Bundesministerium für Atomkernenergie und von der Deutschen Forschungsgemeinschaft durch finanzielle Mittel für Geräte unterstützt.

\title{
Die UV-Absorptionsspektren einiger einfacher Chinoxalin-Derivate
}

\author{
Von H. H. Perkampus \\ Aus dem Institut für Physikalische Chemie und Elektrochemie der Technischen Hochschule Hannover \\ (Z. Naturforschg. 17 a, 614-621 [1962] ; eingegangen am 31. März 1962)

\begin{abstract}
Die UV-Absorptionsspektren 13 einfacher Chinoxalin-Derivate, bei denen die Substituenten keinen
An Hand der Feinstruktur der UV-Absorptionsspektren der 2,3-Di-methoxy- und iso-propoxy-
\end{abstract} \\ Anlaß zu tautomeren Wechselwirkungen geben, wurden in verschiedenen Lösungsmitteln gemessen \\ und diskutiert. \\ Derivate und deren Fluoreszenzspektren sowie der Lösungsmittelabhängigkeit aller Absorptions- \\ spektren wird versucht, eine Zuordnung der langwelligen, überlagerten ${ }^{1} \mathrm{~L}$-Banden zu treffen.
}

Im Zusammenhang mit unseren ir-spektroskopischen Untersuchungen an Chinoxalinen ${ }^{1}$ werden in der vorliegenden Arbeit die UV-Absorptionsspektren einiger Chinoxalin-Derivate mitgeteilt und diskutiert. Chinoxalin selbst wurde im Hinblick auf Untersuchungen über die n- $\tau$-Banden in den UV-Absorptionsspektren von Di-Aza-Verbindungen in letzter Zeit mehrfach untersucht ${ }^{2}$. Unser Interesse gilt daher dem Einfluß von Substituenten auf das Absorptionsspektrum des Chinoxalins, da hierüber, von speziellen Problemstellungen abgesehen ${ }^{3}$, nur wenig Material vorliegt ${ }^{4}$. In dieser Mitteilung sollen zu-

1 H.-H. Perkampus u. A. Roders, Z. Naturforschg. 15 b, 1 [1960].

2 S. F. Mason, J. Chem. Soc., London Spec. Public. No. 3, 139 [1955]. - R. C. Hirt, F. T. King u. J. C. Cavignol, J. Chem. Phys. 25, 514 [1956]. - G. Favini, Nuovo Cim. $\mathrm{X}, \mathbf{8 ,} 60$ [1958]. nächst nur Substituenten berücksichtigt werden, die keinen Anlaß zu Tautomerieerscheinungen geben und bei denen ferner die mesomere Wechselwirkung als gering anzunehmen ist. Die untersuchten Derivate sind in Tab. 1 zusammengestellt, wobei die $\mathrm{Nu}$ merierung der Ringatome in der beim Stammkörper angegebenen Weise erfolgte.

\section{Das Absorptionsspektrum des Chinoxalins}

Die Grundlage einer Diskussion der Absorptionsspektren der Chinoxalin-Derivate bildet das Absorp-

${ }^{3}$ G. W. Cheeseman, J. Chem. Soc. 1958, 108.

${ }^{4}$ Vgl. die Zusammenstellung in J. C. E. Simpsox, Condensed Pyridazin and Pyrazin Rings, Interscience Publishers, New York 1953. 
tionsspektrum des Chinoxalins, das in Abb. 1 für Vergleichszwecke in verschiedenen Lösungsmitteln dargestellt ist. Da Naphthalin der zugehörige aromatische Stammkörper ist, kann das Absorptionsspektrum des Chinoxalins unter gleichzeitiger Berücksichtigung des Chinolin-Spektrums hierauf bezogen werden. Vergleicht man das Chinoxalin mit dem Chinolin, dessen Banden von $Z_{\text {ANKER }}{ }^{5}$ mit Hilfe des Plattschen Modells ${ }^{6}$ zugeordnet wurden, und deren relative Zuordnung durch neuere Untersuchungen über die Fluoreszenzpolarisation von ZimmerManN und Mitarbeitern ${ }^{7}$ sichergestellt ist, so ergibt sich für Chinoxalin eine analoge Zuordnung, wie sie bereits von MüLLER und DöRR vorgenommen worden ist ${ }^{8}$. Danach ist der in $\mathrm{n}$-Heptan strukturierte langwellige $\pi \cdot \pi$-Übergang $\left(v_{0-0}=31600 \mathrm{~cm}^{-1}\right)$ als Überlagerung der ${ }^{1} \mathrm{~L}_{\mathrm{a}}$ - und ${ }^{1} \mathrm{~L}_{\mathrm{b}}$-Banden aufzufassen. Dieser Bande ist der schwächere $n-\pi$-Übergang vor-

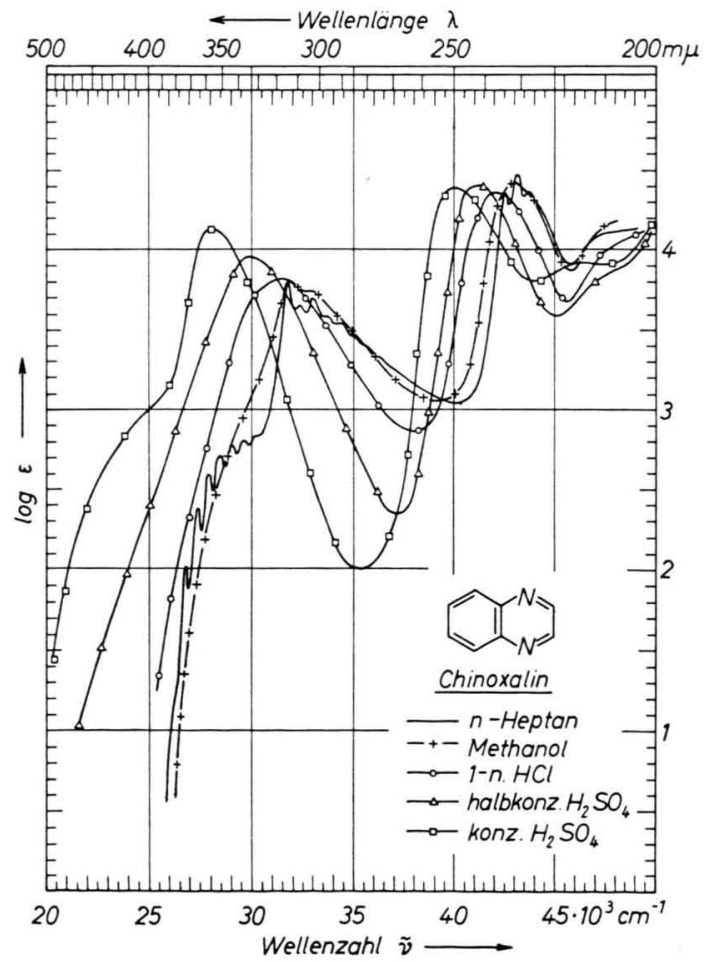

Abb. 1. Absorptionsspektrum des Chinoxalins in verschiedenen Lösungsmitteln bei Raumtemperatur, $c=10^{-4}$-molar.

5 V. Zanker, Z. phys. Chem., N.F. 2, 52 [1954].

${ }_{6}^{6}$ J. R. Platt, J. Chem. Phys. 17, 484 [1949].

7 H. Zimmermann u. N. Joop, Z. Elektrochem. 65, 61 [1961].

8 R. Mürler u. F. Dörr, Z. Elektrochem. 63, 1150 [1959].

${ }^{9}$ R. C. Hirt u. Mitarb., vgl. Anm. 2. - G. Brealey u. M. gelagert, der auf Grund seiner Blauverschiebung beim Übergang von unpolaren zu polaren Lösungsmitteln hinreichend charakterisiert werden kann ${ }^{9}$.

Als intensivste Bande tritt bei $43000 \mathrm{~cm}^{-1}$ die ${ }^{1} B_{\mathrm{b}}$-Bande auf.

Bis zu halbkonzentrierter Schwefelsäure als Lösungsmittel verschieben sich die überlagerten Banden gemeinsam bathochrom, ohne daß eine Auftrennung der beiden Banden erfolgt. Erst in konz. Schwefelsäure erscheint die ${ }^{1} \mathrm{~L}_{\mathrm{a}}$-Bande als Schulter zwischen 26000 und $20000 \mathrm{~cm}^{-1}$. Dieses Absorptionsspektrum ist charakteristisch für das zweifach positive Chinoxalinium-Kation.

\section{Die Absorptionsspektren der Chinoxalin- Derivate}

Neben Chinoxalin zeigen, wie in Tab. 1 durch ein Kreuz vermerkt ist, 2-Methyl-, 2,3-Dimethyl-, 5-Methyl-, 6-Methyl-, 5-Chlor-, 6-Chlor- und 6,7-Dimethyl-chinoxalin eine deutlich als Schulter abgesetzte und z. Tl. strukturierte $n-\pi$-Bande, über deren Lösungsmittelabhängigkeit getrennt berichtet werden soll ${ }^{10}$. Bei den übrigen Derivaten bedingt der stärkere auxochrome Charakter bzw. die gesteigerte Wirksamkeit des Substituenten bei der Substitution im Heterokern, eine Blauverschiebung der $n-\pi$ Bande ${ }^{11}$. Da auch gleichzeitig eine Rotverschiebung der $\pi-\pi$-Banden erfolgt, wird die $n-\pi$-Bande völlig überdeckt. Aus Tab. 1 ist zu ersehen, daß die Rotverschiebung für die oben genannten Derivate, die die $n-\pi$-Banden zeigen, relativ gering ist, während sie für die übrigen Derivate bis auf $2200 \mathrm{~cm}^{-1}$, z. B. beim 2,3-Dibrom-chinoxalin, anwächst.

Der geringe Einfluß der Substituenten bei den genannten Derivaten bewirkt auch keine merkliche Änderung der Intensität dieser Banden gegenüber ihrer Intensität im unsubstituierten Chinoxalin, wie aus Tab. 1, Spalte 4, zu ersehen ist. Die Absorptionsspektren dieser Derivate sind dem Absorptionsspektrum des Chinoxalins, abgesehen von der Feinstruktur in der n- $\tau$-Bande, noch weitgehend ähnlich, so daß in dieser Arbeit von der Darstellung der Absorptionsspektren abgesehen werden kann.

Kasha, J. Amer. Chem. Soc. 77, 4462 [1955]. - H. Mc Connell, J. Chem. Phys. 20, 700 [1952].

10 H.-H. Perkampus u. F. Baucke, auszugsweise vorgetragen auf der Tagung der Deutschen Bunsenges. 1961 in Karlsruhe.

11 F. Halverson u. R. C. Hirt, J. Chem. Phys. 19, 711 [1951]. 


\begin{tabular}{|c|c|c|c|c|c|c|c|c|}
\hline \multirow{2}{*}{$\begin{array}{l}\text { Nr. } \\
\text { der } \\
\text { Abb. }\end{array}$} & \multirow{2}{*}{ Derivat } & \multicolumn{3}{|c|}{${ }^{1} \mathrm{~L}_{\mathrm{b}}$-Bande } & \multirow{2}{*}{$\begin{array}{l}\mathrm{n} \rightarrow \pi- \\
\text { Bande }\end{array}$} & \multicolumn{3}{|c|}{${ }^{1} \mathrm{~B}_{\mathrm{b}}$-Bande } \\
\hline & & $v\left[\mathrm{~cm}^{-1}\right]$ & $\log \varepsilon$ & $\Delta v$ & & $v\left[\mathrm{~cm}^{-1}\right]$ & $\log \varepsilon$ & $\Delta v$ \\
\hline 1 & $\begin{array}{lll}6 & 3 \\
7 & 2 & \\
7 & 2 & \end{array}$ & 31600 & 3,78 & - & + & $\begin{array}{l}42450 \\
43050 \\
43750\end{array}$ & 4,47 & - \\
\hline & 2-Methyl- & 31600 & 3,72 & 0 & + & $\begin{array}{l}42000 \\
42700 \\
43400\end{array}$ & 4,47 & -450 \\
\hline 2 & 2-Chlor- & 30200 & 3,68 & -1400 & $(+)$ & $\begin{array}{l}41300 \\
41950 \\
42530\end{array}$ & 4,53 & -1150 \\
\hline 3 & 2-Methoxy- & 29750 & 3,68 & -1850 & - & $\begin{array}{l}41000 \\
41700 \\
42350\end{array}$ & 4,22 & -1450 \\
\hline & 2,3-Dimethyl- & 31600 & 3,83 & 0 & + & $\begin{array}{l}41900 \\
42550 \\
43150\end{array}$ & 4,49 & -550 \\
\hline 4 & 2,3-Dimethoxy- & 30800 & 4,12 & -1800 & - & 40700 & 4,20 & -1750 \\
\hline 5 & 2,3-Di-isopropoxy- & 30500 & 4,16 & -1100 & - & $\begin{array}{l}40400 \\
41050\end{array}$ & 4,20 & -2050 \\
\hline 6 & 2,3-Dichlor- & 29750 & 3,99 & -1850 & - & $\begin{array}{l}40550 \\
41200 \\
41850\end{array}$ & 4,53 & -1900 \\
\hline 7 & 2,3-Dibrom- & 29450 & 3,95 & -2250 & - & $\begin{array}{l}40000 \\
40750 \\
41300\end{array}$ & 4,58 & -2450 \\
\hline & 5-Methyl- & 31400 & 3,70 & -200 & + & $\begin{array}{l}41600 \\
42200 \\
42750\end{array}$ & 4,53 & -850 \\
\hline & 6-Methyl- & 31200 & 3,72 & -450 & + & $\begin{array}{l}41800 \\
42500 \\
43150\end{array}$ & 4,48 & -650 \\
\hline & 5-Chlor- & 31400 & 3,68 & -200 & + & $\begin{array}{l}41300 \\
42000 \\
42650\end{array}$ & 4,56 & -1150 \\
\hline & 6-Chlor- & 30800 & 3,78 & -850 & + & $\begin{array}{l}41750 \\
42450 \\
43050\end{array}$ & 4,50 & -1700 \\
\hline & 6,7-Dimethyl- & 31150 & 3,87 & -450 & $\cdot+$ & $\begin{array}{l}42550 \\
42000 \\
41400\end{array}$ & 4,52 & -1050 \\
\hline
\end{tabular}

Tab. 1. Lage, Intensität und Zuordnung der Absorptionsbanden einiger Chinoxalin-Derivate. Lösungsmittel n-Hexan, n-Heptan; Temperatur: $20^{\circ} \mathrm{C}$.

Tab. 1 läßt ferner, in Verbindung mit den dargestellten Spektren, erkennen, daß eine Substitution im Heterokern von größerem Einfluß ist als im aromatischen Kern. So bewirkt die Substitution von
Chlor in 2-Stellung (vgl. Abb. 2) eine Rotverschiebung von $1400 \mathrm{~cm}^{-1}$, die beim 2,3-Dichlorderivat (vgl. Abb. 6) auf $1850 \mathrm{~cm}^{-1}$ anwächst.

Innerhalb der untersuchten mono-substituierten 


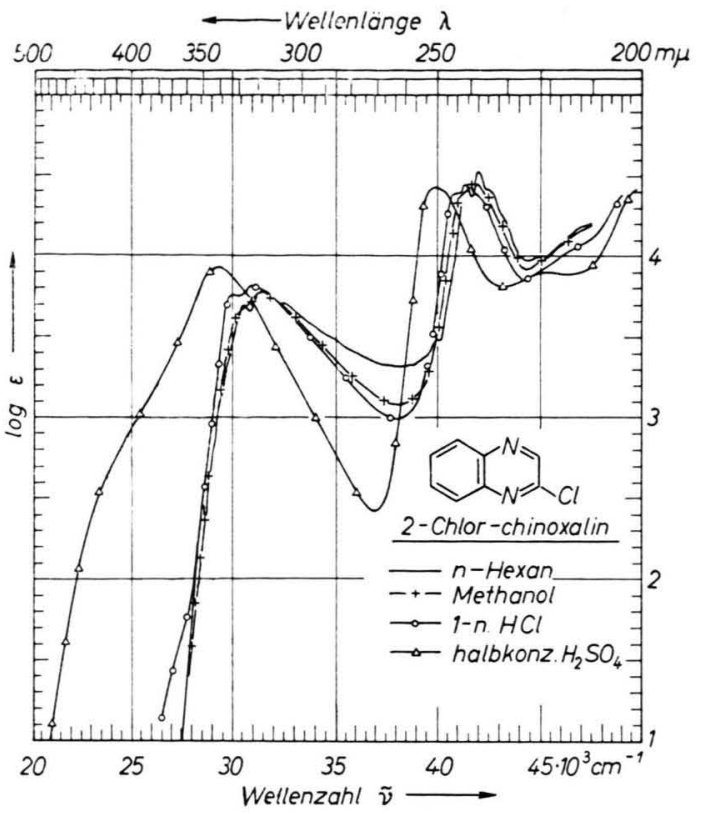

Abb. 2. Absorptionsspektrum des 2-Chlor-chinoxalins in verschiedenen Lösungsmitteln bei Raumtemperatur, $c=10^{-4}$-molar.

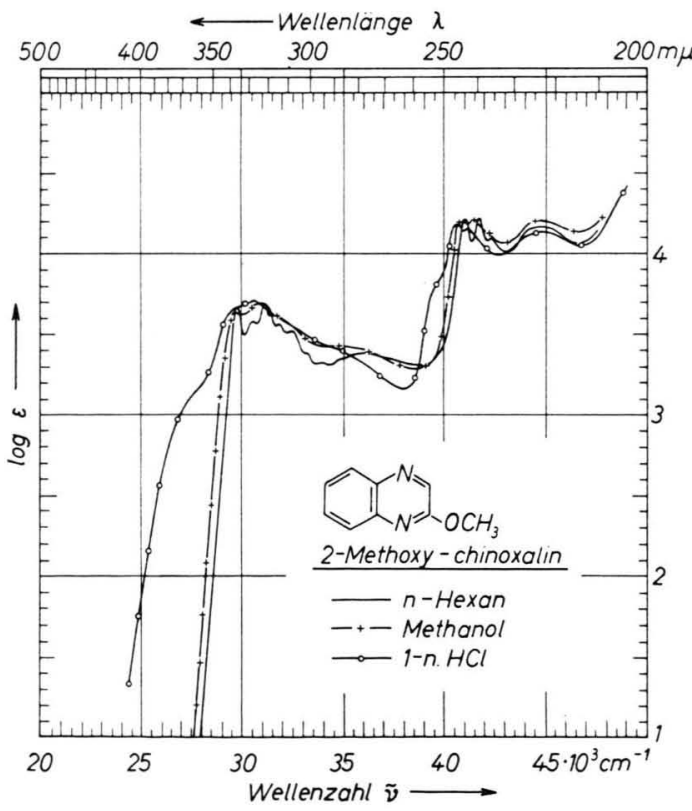

Abb. 3. Absorptionsspektrum des 2-Methoxy-chinoxalins in verschiedenen Lösungsmitteln bei Raumtemperatur, $c=10^{-4}$-molar.
Derivate zeigt das 2-Methoxy-chinoxalin, wie aus Tab. 1 und Abb. 3 zu ersehen ist, den stärksten bathochromen Effekt mit $1850 \mathrm{~cm}^{-1}$, der jedoch beim Übergang zum 2,3-Dimethoxy-chinoxalin nicht weiter anwächst, sondern rückläufig ist, da die Rotverschiebung hier nur noch $800 \mathrm{~cm}^{-1}$ beträgt. Substitution von Halogen im aromatischen Kern bewirkt dagegen eine wesentlich kleinere Rotverschiebung der langwelligen Banden, wie in Tab. 1 die Beispiele 5-Chlor- und 6-Chlor-chinoxalin zeigen, bei denen auch die $n-\tau$-Banden wieder zu beobachten sind.

Nimmt man das langwellige Absorptionsmaximum des 2-Hydroxy-chinoxalins-Anions hinzu, das in 1- $n$. $\mathrm{NaOH}$ bei $28500 \mathrm{~cm}^{-1}$ gemessen wurde ${ }^{12}$, so ergibt sich für Monosubstitution im Heterokern eine gleiche Abstufung der Wirksamkeit, wie sie bei Benzol bekannt ist ${ }^{13}$ :

$$
-\mathrm{CH}_{3}<-\mathrm{Cl}<\mathrm{O}-\mathrm{CH}_{3}<-\mathrm{O}^{-} .
$$

Eine Erweiterung dieser Reihe auf andere auxochrome Gruppen, wie die $\mathrm{OH}$-Gruppe oder die $\mathrm{NH}_{2}$ Gruppe, ist wegen der zusätzlichen innermolekularen Wechselwirkung nicht möglich, so daß sich eine lückenlose Reihe für die bathochrome Wirksamkeit der Substituenten nicht aufstellen läßt.

Bei Betrachtung der Absorptionsspektren fällt besonders auf, daß die Schwingungsstruktur der langwelligen Banden durch einige Substituenten stark begünstigt wird. Dies ist z. B. der Fall beim 2-Methoxy-, 2,3-Dimethoxy und 2,3-Diisopropoxy-chinoxalin, aber in begrenztem Umfang auch bei den 2,3-Dihalogen-chinoxalinen. Wie die Abb. 3-7 erkennen lassen, ist mit der Disubstitution eine Intensitätserhöhung verbunden.

Der gleiche Gang wie in den ${ }^{1} \mathrm{~L}-\mathrm{Banden}$ kann auch bei der ${ }^{1} \mathrm{~B}_{\mathrm{b}}$-Bande in Abhängigkeit vom Substituenten beobachtet werden. Zum Teil sind die Unterschiede größer, jedoch bleibt die relative Abstufung im wesentlichen erhalten, wie die entsprechenden Werte in Tab. 1 erkennen lassen. Bei allen Derivaten sind in der ${ }^{1} \mathrm{~B}_{\mathrm{b}}$-Bande 3 Maxima zu erkennen, deren Abstand im Mittel $700 \mathrm{~cm}^{-1}$ beträgt. Lediglich bei den Alkoxy-Derivaten ist diese Bande breit und strukturlos. Gleichzeitig ist bei diesen Derivaten die Intenstät der ${ }^{1} B_{b}$-Bande stark erniedrigt, während die Intensität der langwelligen ${ }^{1} \mathrm{~L}$-Banden um einen entsprechenden Betrag erhöht ist.

13 M. Pestemer u. D. Brück, in Houben-Weyl, Methoden der organ. Chemie, Bd. 3/2, Thieme-Verlag, Stuttgart 1955. 


\section{Die Feinstruktur der ${ }^{1}$ L-Banden}

Wie bereits erwähnt, tritt in einigen Fällen die Schwingungsstruktur der ${ }^{1} \mathrm{~L}$-Banden in unpolaren Lösungsmitteln bereits bei Raumtemperatur außerordentlich stark in Erscheinung. Während beim Chinoxalin selbst innerhalb der ${ }^{1} \mathrm{~L}$-Banden in n-Heptan nur fünf Maxima gut zu bestimmen sind, sind es beim 2-Methoxy-Derivat sieben (Abb. 3), beim 2,3-Dimethoxy- acht (Abb. 4) und beim 2,3-Di-iso-

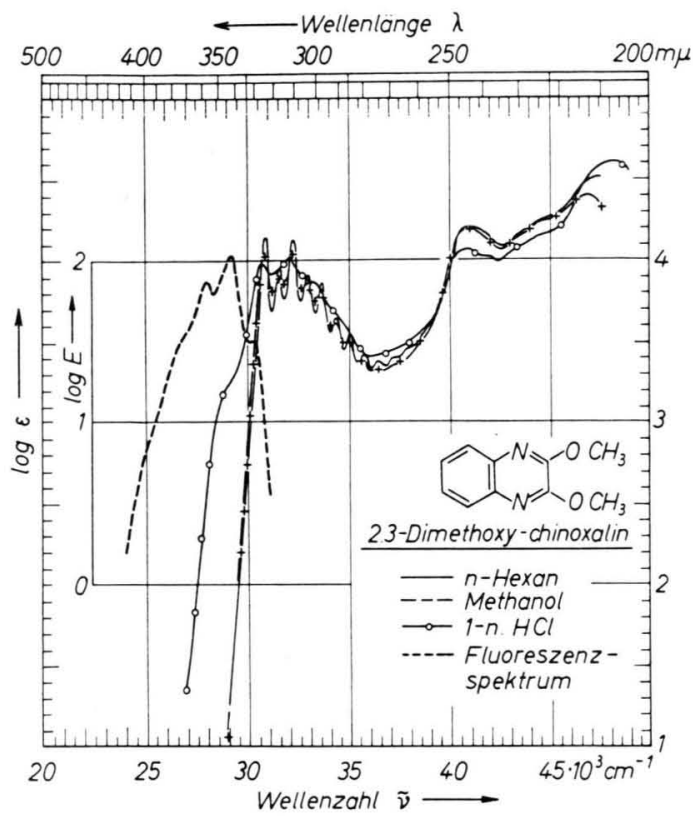

Abb. 4. Absorptionsspektrum des 2,3-Dimethoxy-chinoxalins in verschiedenen Lösungsmitteln bei Raumtemperatur, $c=10^{-4}$-molar. Gestrichelt: Fluoreszenzspektrum in Cyclohexan.

propoxy-chinoxalin zehn (Abb. 5) sicher in der Lage zu bestimmende Maxima, die in Tab. 2 zusammengestellt sind. Außerdem sind die Maxima für 2,3-Dichlor-, 2,3-Dibrom-chinoxalin und Chinolin in der Tabelle mit aufgeführt. Aus dem in den Abb. 3-7 zu ersehenden Intensitätswechsel der Schwingungsstruktur lassen sich zwei Schwingungsquanten ermitteln, wie die Differenzen in Tab. 2 zeigen. Danach tritt bei allen vermessenen Derivaten eine Hauptschwingung mit ca. $1300-1400$ und eine weitere mit $600-700 \mathrm{~cm}^{-1}$ auf. Durch die Überlagerung der ${ }^{1} \mathrm{~L}_{b}$-Bande mit der ${ }^{1} \mathrm{~L}_{\mathrm{a}}$-Bande ist die Zuordnung der Schwingungsquanten zu einem dieser Übergänge zunächst erschwert. Berücksichtigt man jedoch, daß die ${ }^{1} \mathrm{~L}_{\mathrm{a}}$-Bande des Chinolins auch im Tieftemperatur- spektrum kaum Feinstruktur aufweist ${ }^{14}$, so kann man annehmen, daß die Feinstruktur bei diesen Chinoxalinderivaten wie beim Chinolin in $\operatorname{der}{ }^{1} \mathrm{~L}_{b}$ -

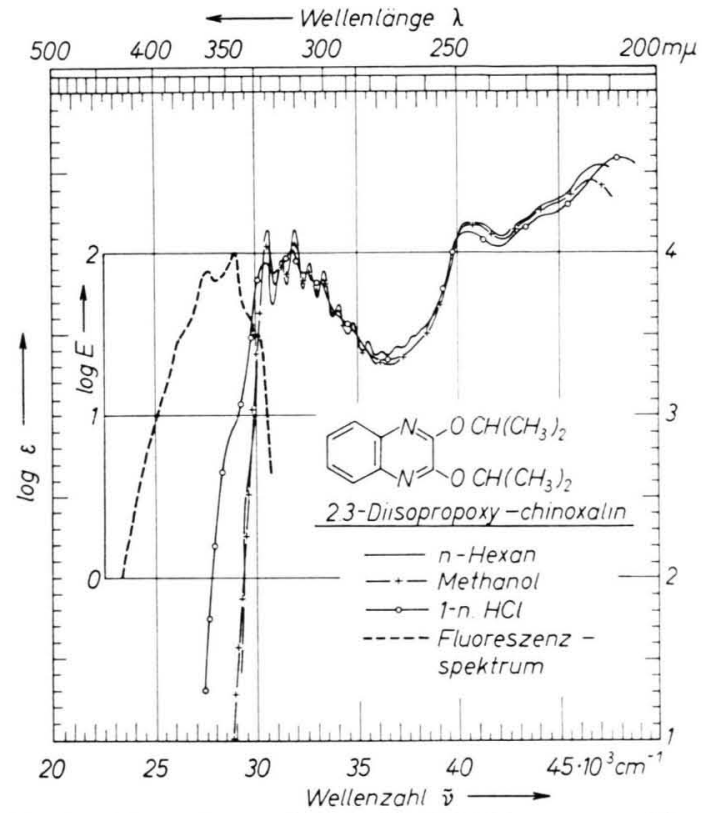

Abb. 5. Absorptionsspektrum des 2,3-Diisopropoxy-chinoxalins in verschiedenen Lösungsmitteln bei Raumtemperatur, $c=10^{-4}$-molar. Gestrichelt: Fluoreszenzspektrum in Cyclo-

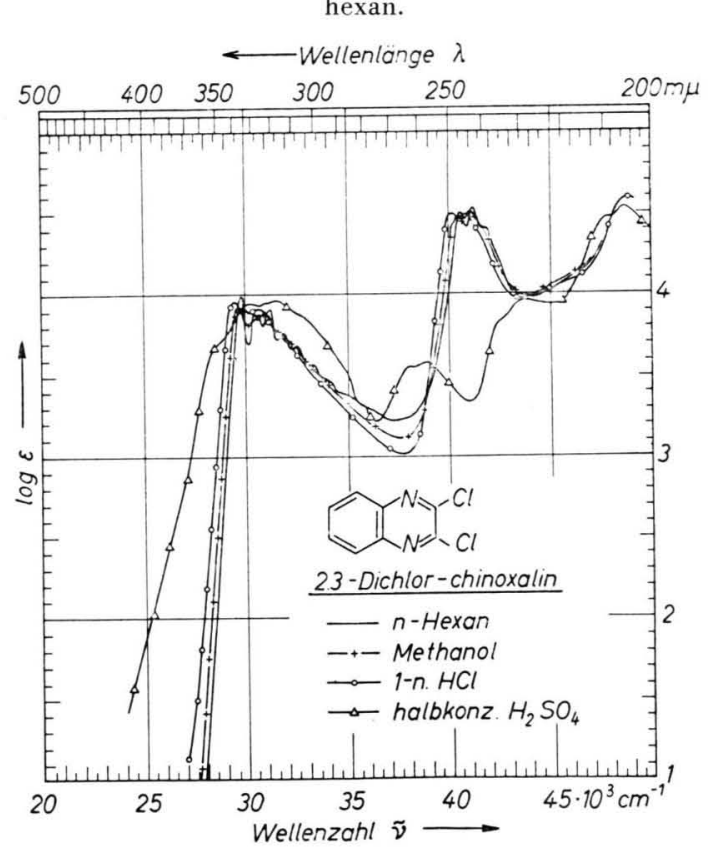

Abb. 6. Absorptionsspektrum des 2,3-Dichlor-chinoxalins in verschiedenen Lösungsmitteln bei Raumtemperatur, $c=10^{-4}$-molar.

14 V. Zanker u. W. Schmid, Chem. Ber. 90, 2253 [1957]. 


\begin{tabular}{|c|c|c|c|c|c|c|c|c|c|c|c|c|c|c|}
\hline \multirow{2}{*}{$\begin{array}{l}\text { Utber- } \\
\text { gang } \\
0-v\end{array}$} & \multicolumn{2}{|c|}{ Chinoxalin } & \multicolumn{2}{|c|}{ 2-Methoxy- } & \multicolumn{2}{|c|}{$\begin{array}{c}2,3- \\
\text { Dimethoxy- }\end{array}$} & \multicolumn{2}{|c|}{$\begin{array}{l}\text { 2,3-Di-iso- } \\
\text { propoxy- }\end{array}$} & \multicolumn{2}{|c|}{ 2,3-Dichlor- } & \multicolumn{2}{|c|}{ 2,3-Dibrom- } & \multicolumn{2}{|c|}{ Chinolin } \\
\hline & $v$ & $\Delta v$ & $v$ & $\Delta v$ & $\Delta v$ & $\Delta v$ & $v$ & $\Delta v$ & $v$ & $\Delta v$ & $v$ & $\Delta v$ & $v$ & $\Delta v$ \\
\hline $0-0$ & $\begin{array}{l}31600 \\
32150\end{array}$ & 1300 & $\begin{array}{l}29750 \\
30500\end{array}$ & 1350 & $\begin{array}{l}30800 \\
31450\end{array}$ & 1400 & $\begin{array}{l}30500 \\
31250\end{array}$ & 1400 & $\begin{array}{l}29750 \\
30300 \\
30550\end{array}$ & 1400 & $\begin{array}{l}29450 \\
30200\end{array}$ & 1500 & $\begin{array}{l}31950 \\
32700\end{array}$ & 1400 \\
\hline $0-1$ & $\begin{array}{l}32900 \\
33500\end{array}$ & 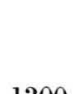 & $\begin{array}{l}31100 \\
31600 \\
31800\end{array}$ & & $\begin{array}{l}32200 \\
32950\end{array}$ & 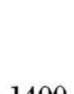 & $\begin{array}{l}31900 \\
32600\end{array}$ & . & $\begin{array}{l}31150 \\
31800\end{array}$ & . & $\begin{array}{l}30950 \\
31700\end{array}$ & & 33350 & \\
\hline $0-2$ & 34200 & 1000 & $\begin{array}{l}32500 \\
33250\end{array}$ & 1400 & 33600 & 1400 & $\begin{array}{l}33300 \\
34050\end{array}$ & 1400 & 32500 & 1350 & & & & \\
\hline $0-3$ & & & & & $\begin{array}{l}34300 \\
35050\end{array}$ & & $\begin{array}{l}34800 \\
35450\end{array}$ & a & & & & & & \\
\hline $0-4$ & & & & & & & $\begin{array}{l}36150 \\
36800\end{array}$ & & & & & & & \\
\hline
\end{tabular}

Tab. 2. Feinstruktur der ${ }^{1} \mathrm{~L}_{b}$-Bande für verschiedene im Heterokern substituierte Chinoxalin-Derivate. Zahlenwerte in $\mathrm{cm}^{-1}$. Lösungsmittel: $\mathrm{n}$-Hexan, $\mathrm{n}$-Heptan; Temperatur: $20^{\circ} \mathrm{C} . \Delta v$ ist die Differenz zwischen den in Spalte 1 bezeichneten Schwingungsübergängen in $\mathrm{cm}^{-1}$.

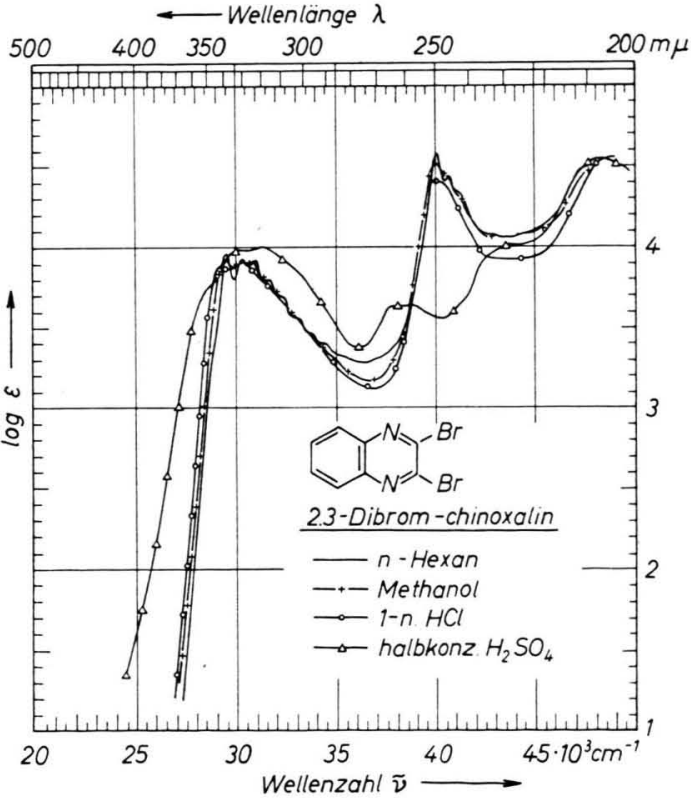

Abb. 7. Absorptionsspektrum des 2,3-Dibrom-chinoxalins in verschiedenen Lösungsmitteln bei Raumtemperatur, $c=10^{-4}$-molar.

Bande zum Ausdruck kommt. Diese Annahme kann durch folgende Argumente gestützt werden:

1. Von allen untersuchten Derivaten zeigt das Absorptionsspektrum des 2-Methoxy-chinoxalins, wie Abb. 3 erkennen läßt, im Bereich von 34000 bis $39000 \mathrm{~cm}^{-1}$ in n-Heptan ein flaches Maximum bei $36000 \mathrm{~cm}^{-1}$, dessen Lage und geringe Struktur der
${ }^{1} \mathrm{~L}_{\mathrm{a}}$-Bande im Chinolin ähnelt und dieser gegenüber eine geringfügige Rotverschiebung aufweist. Durch polare Lösungsmittel wird diese Bande in gleicher Weise rotverschoben wie die entsprechende Bande im Chinolin. In $1-n$. HCl tritt eine weitere Rotverschiebung dieser Bande ein, wie aus Abb. 3 zu erkennen ist, so daß es sich hier sehr wahrscheinlich um die ${ }^{1} \mathrm{~L}_{\mathrm{a}}$-Bande handelt, die wegen der starken Rotverschiebung der ${ }^{1} \mathrm{~L}_{\mathrm{b}}$-Bande $\left(\Delta v=1850 \mathrm{~cm}^{-1}\right)$ sich bei dieser Verbindung deutlicher absetzt als bei den übrigen Derivaten.

Bezieht man daher auf diese Verbindung und vergleicht den Abfall der überlagerten L-Banden zu kurzen Wellen hin, so findet man stets, daß dieser bei allen Chinoxalin-Derivaten zwischen 33000 und $38000 \mathrm{~cm}^{-1}$ in $\mathrm{n}$-Heptan in der Form erfolgt, wie er beim 2-Methoxy-chinoxalin erst in einem polaren Lösungsmittel beobachtet wird, so daß sich in diesem Bereich die ${ }^{1} \mathrm{~L}_{\mathrm{a}}$-Bande allgemein durch Anhebung der Absorption bemerkbar macht.

2. Aus der geschilderten Form der Überlagerung in den L-Banden kann man folgern, daß eine Überlagerung sich erst bei höheren Schwingungsübergängen der ersten ${ }^{1} \mathrm{~L}$-Bande bemerkbar machen sollte. Falls daher die Schwingungsquanten der zweiten ${ }^{1}$ L-Bande sich denen der ersten überlagerten, sollte man für die Maxima bei größeren Wellenzahlen Abweichungen von den alternierenden Differenzen 1400 und $700 \mathrm{~cm}^{-1} \mathrm{bzw}$. zusätzliche Differenzen erwarten. Wie Tab. 2 erkennen läßt, ist dies jedoch innerhalb 
der Meßgenauigkeit nicht zu beobachten, was besonders deutlich beim 2,3-Dimethoxy- und 2,3-Di-isopropoxy-chinoxalin zum Ausdruck kommt, die bis 35750 bzw. $36800 \mathrm{~cm}^{-1}$ vermessen worden sind. Obwohl bei diesen Derivaten eine starke UUberlagerung der beiden $\mathrm{L}$-Banden vorliegen muß, wie die Spektren in den Abb. 4 und 5 erkennen lassen, bedeutet dies demnach, daß eine dieser Banden praktisch unstrukturiert sein $\mathrm{muß}$.

3. Daß der genannte Bereich der ${ }^{1} \mathrm{~L}_{\mathrm{a}}$-Bande zuzuordnen ist, zeigt sich ferner bei der Betrachtung der Spektren in saurer Lösung, im Vergleich zu denen in n-Heptan und polaren Lösungsmitteln. Bei der Anlagerung des ersten Protons (etwa in 1-n. $\mathrm{HCl}$ ) erfolgt unter der Voraussetzung, daß das Übergangsmoment der ${ }^{1} \mathrm{~L}_{\mathrm{a}}$-Bande des Chinoxalins wie beim Naphthalin und Chinolin in der kurzen Molekelachse orientiert ist ${ }^{15}$, eine energetische Begünstigung dieses Überganges, so daß sich dieser Übergang stärker als der ${ }^{1} \mathrm{~L}_{\mathrm{b}}$-Übergang bathochrom verschiebt. Dies macht sich in einer geringfügigen Rotverschiebung der überlagerten Banden, aber zugleich sehr viel ausgeprägter in der um $36000 \mathrm{~cm}^{-1}$ stark verminderten Intensität bemerkbar. Noch deutlicher werden diese Verhältnisse in halbkonz. bzw. konz. Schwefelsäure, wo schließlich das zweifach positive Chinoxalinium-Kation vorliegt. Die starke Intensitätsabnahme bei der Protonenanlagerung in diesem Bereich deutet also ebenfalls darauf hin, daß an dieser Stelle eine Absorptionsbande im neutralen Molekül liegt, die auf Grund der beobachteten Rotverschiebung in Analogie zum Chinolin als ${ }^{1} \mathrm{~L}_{\mathrm{a}}$-Bande bezeichnet werden kann.

Von den in Tab. 1 zusammengestellten Chinoxalin-Derivaten zeigen das 2,3-Dimethoxy- und 2,3-Diisopropoxy-chinoxalin eine gut meßbare Fluoreszenz. Wie die Absorptionsspektren dieser Derivate sind auch die Fluoreszenzspektren bei Raumtemperatur strukturiert. Die Struktur läßt sicher ein Hauptschwingungsquant von $1300 \mathrm{~cm}^{-1}$ erkennen, das erwartungsgemäß kleiner ist als das aus dem Absorptionsspektrum ermittelte und für den Grundzustand verbindlich ist. Die Ermittlung dieses Schwingungsquantes ist etwas ungenauer als das aus dem Absorptionsspektrum ermittelte, da der $0-0$-Übergang im Fluoreszenzspektrum infolge der Reabsorption nur als Schulter erscheint. Beide 0-0-Übergänge,

15 J. R. Platt ${ }^{6}$. - V. Z AnKeR $^{14}$. - Vgl. auch C. SANDORFY, Die Elektronenspektren, Verlag Chemie, Weinheim 1961, S. $145 \mathrm{ff}$. in Absorption und Fluoreszenz, liegen etwa $300 \mathrm{~cm}^{-1}$ auseinander, so daß sich das Fluoreszenzspektrum spiegelbildlich an das Absorptionsspektrum anschließt, wie aus den Abb. 4 und 5 hervorgeht, in denen die Fluoreszenzspektren mit eingezeichnet sind. Diese Spiegelbildsymmetrie dürfte daher eine weitere Stütze für die getroffene Zuordnung der strukturierten langwelligen Bande als ${ }^{1} \mathrm{~L}_{\mathrm{b}}$-Bande sein.

Die Aufnahme der Fluoreszenzspektren in polaren Lösungsmitteln ergab keine Verschiebung der Fluoreszenzspektren gegenüber den in Hexan bzw. Cyclohexan vermessenen, so daß bei diesen Derivaten keine wesentliche Änderung des Dipolmomentes im angeregten Zustand gegenüber dem des Grundzustandes anzunehmen ist ${ }^{16}$. Diese Frage war von Interesse, da die beiden fluoreszierenden Derivate eine starke Intensitätszunahme in den ${ }^{1} \mathrm{~L}$-Banden zeigten. Wenn man eine reine Überlagerung der beiden ${ }^{1} \mathrm{~L}$-Banden annimmt, indem man z. B. die Werte der Extinktionskoeffizienten für die ${ }^{1} \mathrm{~L}_{b}$ - und die ${ }^{1} \mathrm{~L}_{\mathrm{a}}$-Bande des 2-Methoxy-chinoxalins zugrunde legt, müßte die Intensität dieser Banden noch unter $\log \varepsilon=3,9$ liegen. Es ist daher anzunehmen, daß für die erhöhte Intensität der langwelligen Bande dieser Derivate $(\log \varepsilon=4,15)$ eine das Übergangsmoment vergrößernde angeregte Grenzstruktur nach (a) bzw. (b) zu berücksichtigen ist:
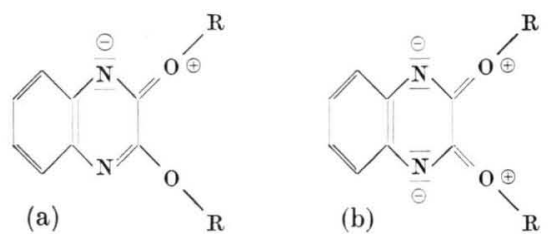

$\mathrm{Da} \beta$ derartige angeregte polare Strukturen keine Wechselwirkung mit polaren Lösungsmittelmolekeln eingehen, kann auf eine sterische Behinderung durch die Alkoxygruppen zurückgeführt werden.

\section{Experimenteller Teil}

Die UV-Absorptionsspektren wurden mit dem ZeissSpektralphotometer PMQ II, das mit einer Hundertpunktautomatik ausgerüstet ist, gemessen. Die Konzentration der Lösungen war $10^{-4}-m . ;$ die Schichtdicken der Küvetten wurden je nach Extinktion zwischen 1, 0,5 und $0,1 \mathrm{~cm}$ variiert.

16 E. Lippert, Z. Elektrochem. 61, 962 [1957]. 
Für die Fluoreszenzspektren wurde eine bereits früher beschriebene Apparatur benutzt ${ }^{17}$, die in der üblichen $45^{\circ}$-Anordnung arbeitet. Die Apparatur wurde im Bereich von 19000 bis $30500 \mathrm{~cm}^{-1}$ mit Hilfe des von LipPerT ${ }^{18}$ normierten Fluoreszenzspektrums des $\beta$. Naphthols in Standardacetatlösung geeicht.. In den Abb. 4 und 5 wurden die auf eins normierten relativen Energieeinheiten im logarithmischen Maßstab dargestellt.

17 H.-H. Perkampus, Z. phys. Chem., N.F. 24, 1 [1960].

18 E. Lippert, N. Nägele, I. Seibold-Blankenstein, H. Staiger u. W. Voss, Z. analyt. Chem. 170, 1 [1959].
Auf die Darstellung der Substanzen wurde im Rahmen einer anderen Arbeit bereits hingewiesen ${ }^{\mathbf{1 9}}$.

Dem Institutsdirektor, Herrn Prof. Dr. Dr. h.c. R. Sunrmann, danke ich für das Interesse an diesen Untersuchungen. Der Deutschen Forschungsgemeinschaft und dem Verband der Chemischen Industrie möchte ich für die Unterstützung dieser Arbeit danken. Herrn Dipl.Chem. F. BAUcke danke ich für die sorgfältige Ausführung der Absorptionsmessungen.

19 Vgl. Anm. ${ }^{1}$ sowie A. Roders, Diplomarbeit Hannover 1959; J. C. E. Simpson ${ }^{4}$.

\title{
Zur Theorie des Einflusses von Lösungsmitteln auf die Elektronenspektren der Moleküle
}

\author{
Von L. Bilor und A. KawsKi \\ Aus dem Physikalischen Institut der Pädagogischen Hochschule, Gdańsk (Polen) * \\ (Z. Naturforschg. 17 a, 621-627 [1962] ; eingegangen am 19. April 1962)
}

\begin{abstract}
Auf Grund der quantenmechanischen Störungsrechnung und des ONsager-Modells für flüssige Medien wird eine Gleichung abgeleitet, die den elektrostatischen Beitrag zur Verschiebung der 0-0-Banden im Absorptions- und Emissionsspektrum des Moleküls mit dem Brechungsindex und mit der Dielektrizitätskonstante der Lösung verbindet. Die Gleichung ermöglicht unter gewissen Voraussetzungen die Bestimmung des Dipolmomentes des ersten angeregten Singulettzustandes der fluoreszierenden Moleküle. Im Sonderfall, wenn die Polarisierbarkeit des gelösten Moleküls zu vernachlässigen ist, erhält man die von Lippert hergeleitete Formel. Die mit der von uns erhaltenen Gleichung aus den Messungen von Lippert bestimmten Dipolmomente liegen denen von Czekalla bedeutend näher.
\end{abstract}

Der Lösungsmitteleinfluß auf die Verschiebung der Absorptions- und Fluoreszenzspektren wurde in vielen Arbeiten ${ }^{1-9}$ behandelt.

Den ersten Vorschlag einer qualitativen Erklärung der Verschiebung von Absorptions- und Fluoreszenzbanden gab JABLoŃski ${ }^{1}$, indem er den Begriff des Fluoreszenzzentrums einführte und dieses für die Absorption und Fluoreszenz der Farbstoffmoleküle in der Lösung verantwortlich machte. Die potentielle Energie des Fluoreszenzzentrums hängt sowohl von der Konfiguration der gelösten und der Lösungsmittelmoleküle als auch vom Anregungszustand des Zentrums ab. Den Elektronenzuständen - dem Grund- und Anregungszustand - entsprechen ver-

\footnotetext{
* Katedra Fizyki, Wyzsza Szkola Pedagogiczna, GdańskWrzeszcz.

1 A. Jablonskr, Z. Phys. 73, 460 [1931].

2 Th. Förster, Z. Elektrochem. 54, 571 [1939].

3 N. S. Bayliss, J. Chem. Phys. 18, 292 [1950].

4 Y. Ooshika, J. Phys. Soc., Japan 9, 594. [1954].
}

schiedene Gleichgewichtskonfigurationen der umgebenden Moleküle. Mit diesen Konfigurationen sind verschiedene Werte der Wechselwirkungsenergie verbunden. Die Absorption erfolgt aus einem dem Grundzustand des gelösten Moleküls entsprechenden Gleichgewichtszustand und führt das System in den FrancK-Condon-Anregungszustand. Die Reorientierungsgeschwindigkeit der Lösungsmittelmoleküle ist im allgemeinen so groß, daß die Fluoreszenz aus dem dem angeregten Molekül entsprechenden Gleichgewichtszustand erfolgt und das System zum Franck-Condon-Grundzustand führt.

Ein wesentlicher Fortschritt in der quantitativen Erklärung der 0-0-Banden-Verschiebung für Mo-

5 E. Lippert, Z. Naturforschg. 10 a, 541 [1955].

6 E. Lippert, Z. Elektrochem. 61, 962 [1957].

7 N. Mataga, Y. Kaifu u. M. Kolzumi, Bull. Chem. Soc., Japan 29, 465 [1956].

8 E. G. McRae, J. Phys. Chem. 61, 562 [1957].

9 N. G. Bachschiew, Opt. i Spektr. 10, 717 [1961]. 